\title{
The cytotoxic effects of ciprofloxacin in Giardia lamblia trophozoites
}

\author{
M.C. Sousa*, J. Poiares-da-Silva \\ Laboratory of Microbiology and Parasitology and Centre of Pharmaceutical Studies, Faculty of Pharmacy, \\ University of Coimbra, 3030 Coimbra, Portugal
}

\begin{abstract}
The cytotoxicity of ciprofloxacin was investigated in culture of Giardia lamblia (ATCC 30957), used as an in vitro cellular model, on the basis of cell growth, morphology, viability, adherence and metabolic studies. The effects on cell membrane integrity were evaluated by permeability of the cells to trypan blue, and the morphological alterations were studied by optical and transmission electronic microscopy. The metabolic studies were performed by measured oxygen uptake with a Clark-type oxygen electrode. Our data show that ciprofloxacin induces loss of viability with morphological alterations and loss of membrane integrity. Ultrastructural observations revealed that this drug promoted modifications on the cell shape, pronounced dorsal vesiculation, plasma membrane blebbing, disaggregation of ribosome, coagulation of cytoplasmic matrix, and heavy deposit of electron-dense precipitates on the cytoplasm and nucleus. Ciprofloxacin also inhibits parasite growth, adherence and $\mathrm{O}_{2}$ uptake in a concentrationdependent manner. Some of these observations suggest that cytotoxicity of ciprofloxacin results in cell death by a necrosis process. We demonstrate that ciprofloxacin has a lethal effect in $G$. lamblia trophozoites and could be used as an alternative drug in giardiasis treatment, particularly in infections that are resistant to other antibiotics. (C) 2001 Elsevier Science Ltd. All rights reserved.
\end{abstract}

Keywords: Giardia lamblia; Ciprofloxacin; $\mathrm{O}_{2}$ uptake; Ultrastructure; Viability; Adherence

\section{Introduction}

Giardia lamblia is a binucleated flagellated protozoan that causes infection of the small intestine that may give rise to diarrhoea with or without malabsorption. This parasite is endemic throughout the world and it is one of the most frequently found intestinal parasites in children living in developing countries. Although various drugs have been available for several decades to treat this infection, none is entirely satisfactory due to the high incidence of undesirable side-effects and a significant failure rate in clearing parasites from the gastrointestinal tract. Some evidence suggests that drug resistance may be responsible for these failures (Kulda and Nohyhová, 1995; Barat and Bloland, 1997). Therefore, there is an obvious need for alternative antigiardial agents.

Abbreviations; CPX, ciprofloxacin; HBSS, Hanks' balanced salt solution; DNA, deoxyribonucleic acid.

* Corresponding author. Tel.: +351-239852567; fax: +351239852569.

E-mail address: mcsousa@ci.uc.pt (M.C. Sousa).
The fluoroquinolone antibiotics such as ciprofloxacin have excellent activity in vitro against bacterial pathogens of the gastrointestinal tract including Escherichia coli, Salmonella spp., Shigella spp., Yersinia enterocolítica, Campylobacter jejuni and Vibrio spp. Bacterial DNA gyrase has been identified as the primary target for quinolone (Kidwai et al., 1998). However, the understanding of the molecular mechanisms leading to loss of bacterial viability remains incomplete. Some investigators have suggested that quinolones induce membrane permeability changes and that these lead to loss of integrity, leakage of cytoplasmic constituents, and cell death (Dougherty and Saukkonen, 1985; Elliot et al., 1987).

Ikerd and Koletar (1993), using a macrodilution method in a semi-solid media, reported antigiardial activity of fluoroquinolones and macrolides.

These findings prompt us to study in vitro effects of ciprofloxacin (CPX) on the cell growth, viability, adherence and morphology of the parasite to evaluate its potential value in chemotherapy of giardiasis and to investigate the drug action mechanism(s). 


\section{Materials and methods}

\subsection{Chemicals}

Bile bovine, L-cysteine, L-ascorbic acid and ferric ammonium citrate were obtained from Sigma Chemical Corp., casitone and yeast extract were obtained from Difco Laboratories, and bovine serum and antibiotic solution were obtained from Biochrom K.G. We are indebted to Bayer for the gift of ciprofloxacin.

\subsection{Parasites and cultures}

Giardia lamblia [WB strain (ATCC 30957) originally from a patient with chronic diarrhoea] was obtained from The American Type Collection, Rockville, MD, USA. Trophozoites were maintained in axenic culture at $37^{\circ} \mathrm{C}$ in $10 \mathrm{ml}$ Diamond's TYI-S-33 medium as modified by Keister (1983), in screw-cap cell culture vials. Penicillin $\mathrm{G}(250 \mu \mathrm{g} / \mathrm{ml})$, streptomycin sulfate $(250 \mu \mathrm{g} / \mathrm{ml})$, gentamicin sulfate $(50 \mu \mathrm{g} / \mathrm{ml})$ and amphotericin B $(0.25$ $\mu \mathrm{g} / \mathrm{ml})$ were added during routine culture. After 2 days, the cultures were harvested by cooling the culture vials at $4{ }^{\circ} \mathrm{C}$ for $15 \mathrm{~min}$ and centrifugation at $1200 \mathrm{~g}$ for 10 min. Trophozoites were washed three times in Hanks' balanced salt solution (HBSS; pH 7.1) and cells were counted in a haemocytometer (Neubaeur cell-counter chamber). These cells were used as inoculum to study the effects of CPX on cell growth, viability, morphology and adherence of $G$. lamblia trophozoites.

\subsection{CPX toxicity studies}

An inoculum of $10^{6}$ trophozoites was exposed to ciprofloxacin $(0.1-2 \mathrm{mg} / \mathrm{ml})$ in HBSS (pH 7.1) for $2 \mathrm{~h}$, at $37^{\circ} \mathrm{C}$, using $10 \mathrm{ml}$ polystyrene screw-capped vials. Control experiments were performed under similar experimental conditions without the drug, in the presence of only the drug solvent (HBSS, pH 7.1). After incubation, the vials were cooled at $4^{\circ} \mathrm{C}$ and the suspension centrifuged at $1200 \mathrm{~g}$ for $10 \mathrm{~min}$. Trophozoites were washed three times in cold HBSS and the cell pellets were resuspended in HBSS. Trophozoite viability was directly determined by phase-contrast microscopy, counting the live and dead cells in a haemocytometer (Hill et al., 1986). Parasites were called viable if they had a characteristic pear-shaped structure, flagellar motility, normal architecture of ventral disc and refractory quality. Viability of $G$. lamblia was also determined by measure of the oxygen uptake by the trophozoites as previously described (Sousa and Poiares-da-Silva, 1999a). The effect of CPX was determined by comparing respiratory rates in control cells and in cells preexposed to the drug. To assess cell membrane integrity, trophozoite suspensions were incubated on ice with an equal volume of $0.4 \%$ trypan blue, and loaded into a haemocytometer for viable cell counting (Aley et al., 1994). The experiments were performed three times and the results were expressed as a percentage of viability.

\subsection{Growth inhibition assay}

The susceptibility of $G$. lamblia growth in vitro to drugs was determined as previously described (Edlind and Hang, 1990). Cultures of log-phase organisms were exposed to CPX at various concentrations in fresh
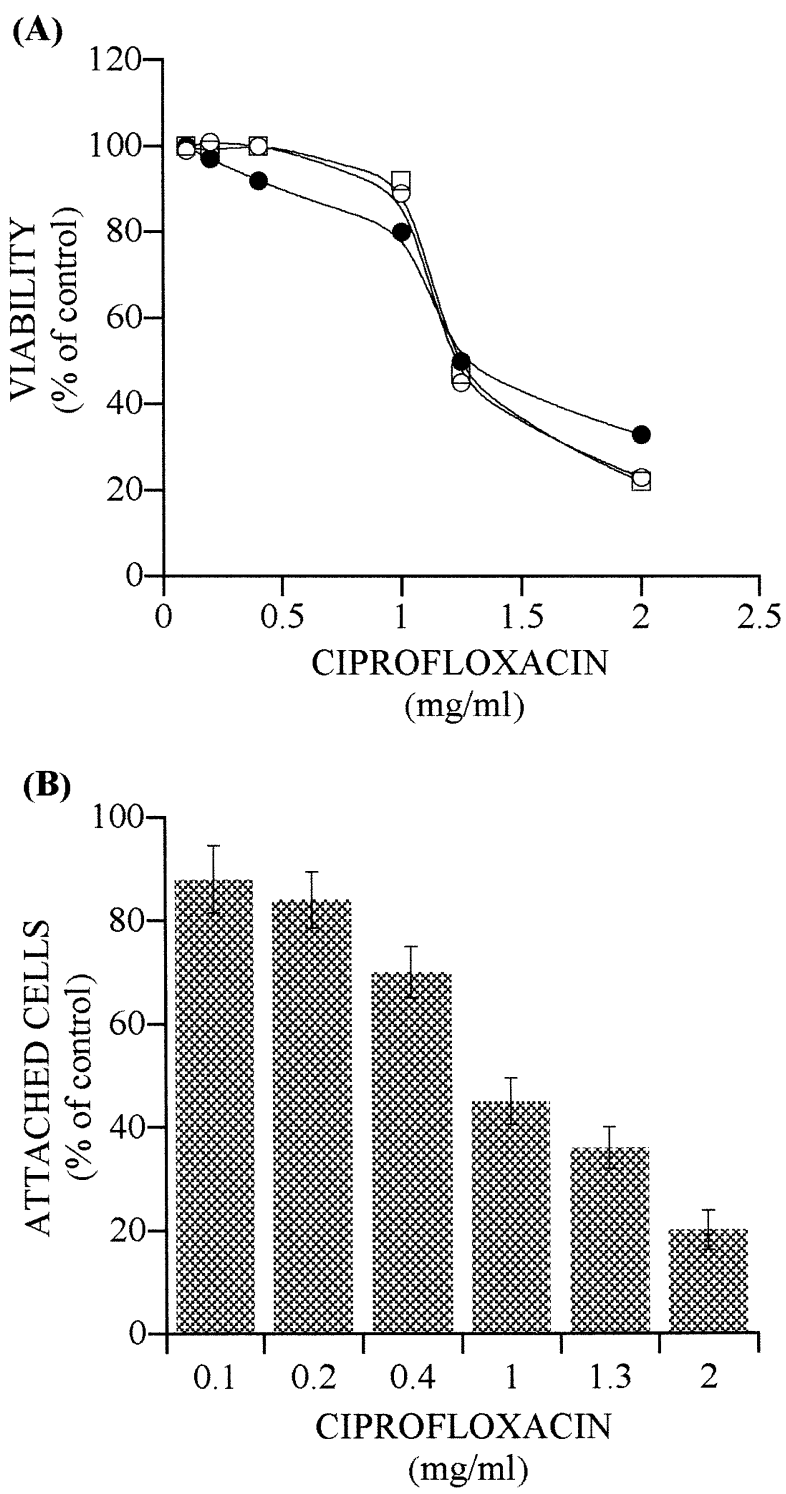

Fig. 1. (A) Killing of Giardia lamblia trophozoites by ciprofloxacin. Trophozoites were incubated with various concentrations of CPX for $2 \mathrm{~h}$ at $37^{\circ} \mathrm{C}$, and trophozoites viability was determined by trypan blue exclusion $(\square)$, morphology $(\bigcirc)$ and $\mathrm{O}_{2}$ uptake $(\bullet)$. These doseresponse curves are typical of three independent experiments and error bars are not indicated since, for most points, they are encompassed by the size of the symbols. (B) Detachment of trophozoites from the culture tubes after $2 \mathrm{~h}$ at $37^{\circ} \mathrm{C}$ in the presence of ciprofloxacin. Standard errors of the mean (vertical bars) were calculated from data of three experiments. 
medium for $48 \mathrm{~h}$. Cell numbers, determined with a haemocytometer, were expressed as a percentage of the cell number in control cultures.

\subsection{Adherence study}

The direct effect of CPX on adherence of G. lamblia trophozoites was evaluated as previously described by Edlind and Hang (1990). An inoculum of $5 \times 10^{5}$ cells was distributed into vials $(10 \mathrm{ml})$ and incubated for $2 \mathrm{~h}$ at $37^{\circ} \mathrm{C}$, in TYI-S-33 medium without antibiotics. Preliminary studies showed that the maximum number of attached cells was attained at this time. The medium with unattached cells was discarded and replaced with prewarmed medium, with and without ciprofloxacin $(0.1-2 \mathrm{mg} / \mathrm{ml})$, and the cultures were incubated for $2 \mathrm{~h}$ at $37^{\circ} \mathrm{C}$. Adherent cells were dislodged by a $10-\mathrm{min}$ incubation in ice-cold HBSS, and the number of attached cells was determined microscopically using a haemocytometer. The experiments were performed three times and the results were expressed as number of attached cells (percentage of control).

\subsection{Transmission electron microscopy}

Samples were treated as reported previously (Sousa and Poiares-da-Silva, 1999b). Cell pellets were fixed with $2.5 \%$ glutaraldehyde in $0.1 \mathrm{~m}$ sodium cacodylate buffer ( $\mathrm{pH} 7.0$ ), post fixed in $1 \%$ osmium tetroxide and uranyl acetate, dehydrated in ethanol and in propylene oxide and embedded in Epon 812 (TAAB 812 resin). Ultrathin sections were stained with lead citrate and uranyl acetate and observed in a JOEL $100 \mathrm{~S}$ electron microscope.

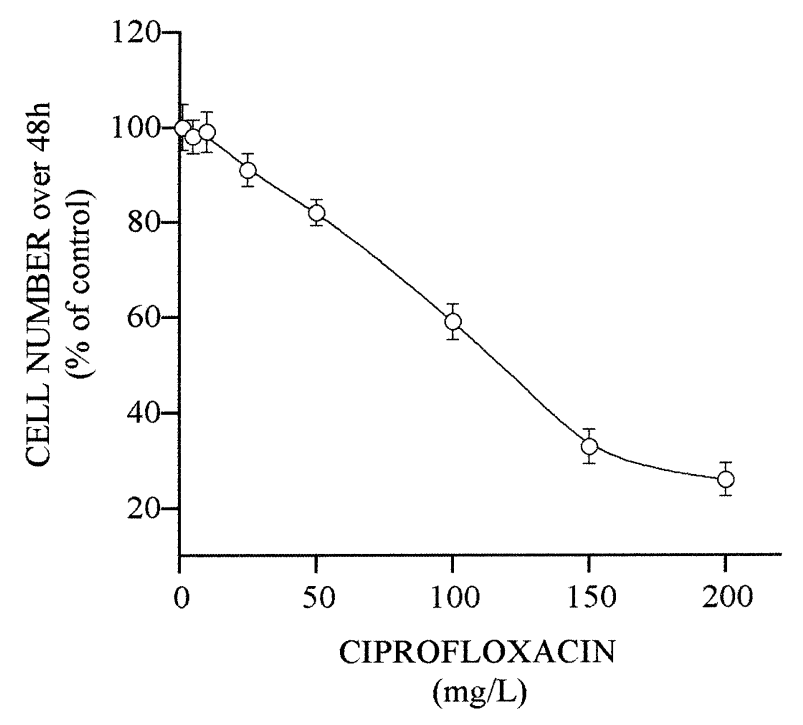

Fig. 2. Inhibition of G. lamblia growth in vitro over $48 \mathrm{~h}$ by ciprofloxacin. Standard errors of the mean (vertical bars) were calculated from data of three experiments.

\section{Results}

\subsection{Viability studies}

CPX induces loss of cellular viability. The doseresponse curves obtained for CPX determined by oxygen uptake and those obtained by morphological and membrane integrity criteria are shown in Fig. 1(A). These results are expressed as the percentage of inhibition of the control. The CPX concentration, which altered the morphology of $19-78 \%$ of the cells, inhibited oxygen uptake by $20-78 \%$ of trophozoites and changed the membrane integrity of $10-79 \%$ of cells. The morphological changes in trophozoites, by phase-contrast microscopy observation, included loss of refractory quality and the presence of numerous precipitates in the nucleus, cytoplasm and plasma membrane (not shown).

\subsection{Adherence inhibition}

G. lamblia trophozoites can attach to in vitro cultured cells as well as to glass and to a variety of artificial substrates (Kulda and Nohynková, 1995). Considering that inhibition of its attachment may have therapeutic potential, it was of interest to study the ability of CPX to promote the G. lamblia detachment. Figure 1(B) shows that G. lamblia attachment to plastic culture vials is strongly inhibited by CPX.

\subsection{Inhibition of growth in vitro}

G. lamblia growth in vitro over $48 \mathrm{~h}$ in the presence of different drug concentrations was measured and expressed as a percentage of the growth in untreated cultures. Our data showed the inhibition of $G$. lamblia growth by ciprofloxacin (Fig. 2).

\subsection{Ultrastructural effects of CPX on trophozoites}

Transmission electron microscopy of untreated $G$. lamblia trophozoites shows that these cells have a typical cytoskeleton with four pairs of flagella and adhesive disc, nucleus, and cytoplasm membrane (Plate 1a). CPX induces morphological changes in cells after exposure for $2 \mathrm{~h}$ at $37^{\circ} \mathrm{C}$. Trophozoites show electron-dense deposits in the nucleus and cytoplasm, plasma membrane blebbing and abnormal shape of the nucleus (Plate 1b). Notice the absence of intact ribosome and cytoplasm precipitation resulting in the appearance of dense blocks (Plate 1b,c). The surface vesicles seem to be associated with the disintegration of the plasma membrane (Plate $1 b, c)$. The empty spaces that appear in the cytoplasm indicate that digestion of intracellular material is occurring in the presence of CPX. In some cells we see displacement of cytoskeletal structures, namely the axonemal microtubules that appear dispersed in the cytoplasm (Plate 1c). 

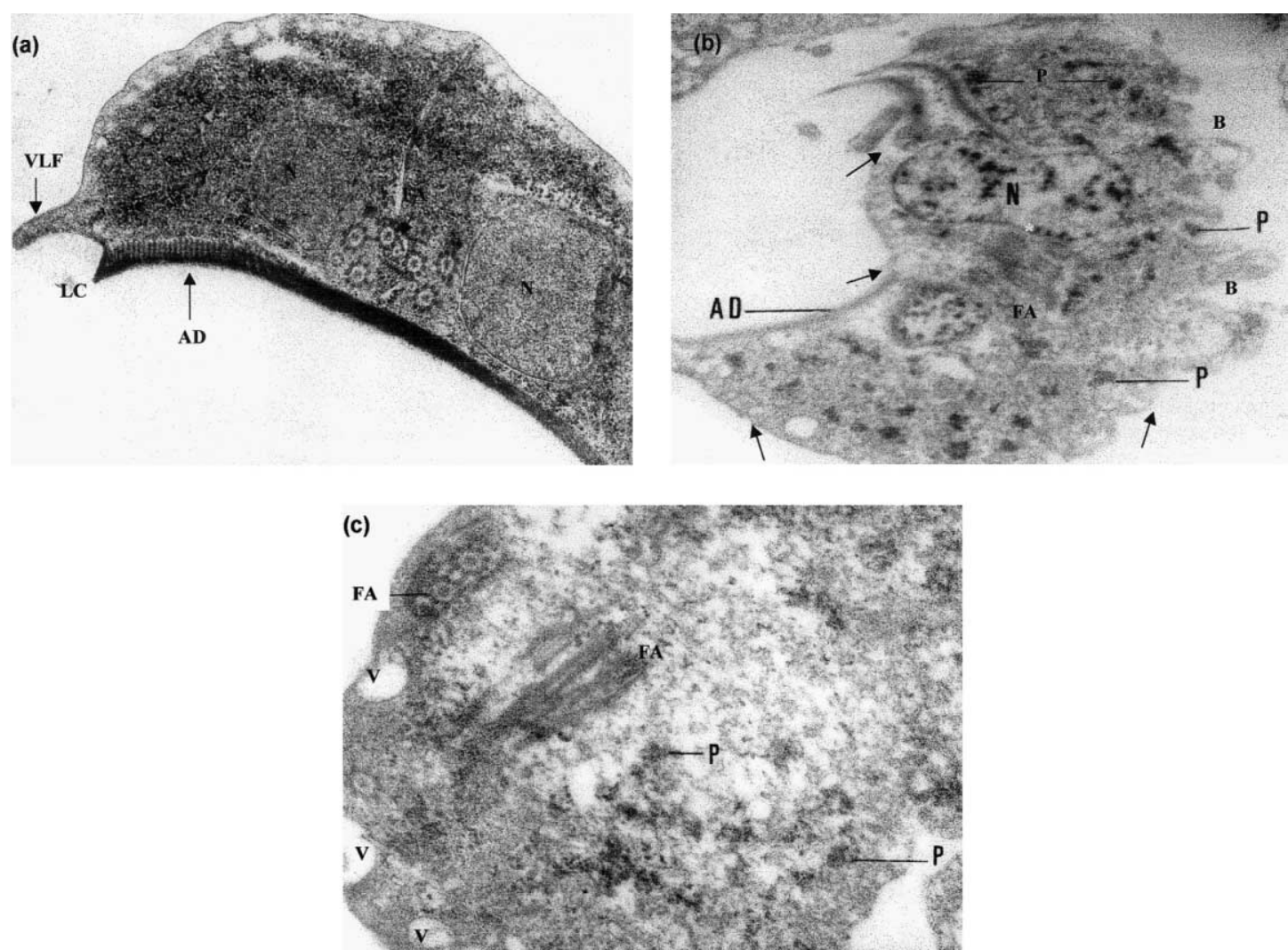

Plate 1. Transmission electron micrographs of G. lamblia trophozoites (a) Untreated parasites $(\times 20,000)$; $(\mathrm{b}, \mathrm{c})$ trophozoites exposed to CPX for $2 \mathrm{~h}$, at $37^{\circ} \mathrm{C}$. (b) Note the electron-dense blocks in nucleus and cytoplasm (P), distortion of nucleus morphology and plasma membrane blebs (B) $(\times 15,000)$. (c) Various dorsal vesicles $(\mathrm{V})$ seem to be associated with plasma membrane rupture (arrowheads), displacement of axonemal microtubules (FA), electron-dense precipitates $(\mathrm{P})$ and empty spaces in cytoplasm $(\times 48,000)(\mathrm{N}$, nucleus; AD, adhesive disc; FA, axonemal microtubules; VLF, ventrolateral flange; LC, lateral crest).

\section{Discussion}

One of the objectives of this work was to evaluate the potential value of ciprofloxacin in chemotherapy of giardiasis. Adherence of G. lamblia trophozoites to enterocytes is essential for colonisation and is considered to play a crucial role in the pathogenesis of giardiasis (Farthing, 1997). Therefore, drugs that inhibit parasite attachment and/or growth have therapeutic potential. Our experimental data showed in vitro cell growth inhibition of G. lamblia trophozoites by ciprofloxacin. CPX caused significant decreases in cell number at $100 \mathrm{mg} / \mathrm{l}$ over $48 \mathrm{~h}$, which is concordant with the results of a previous study (Ikerd and Koletar, 1993). When compared to the action of metronidazole which is considered a "gold standard" antibiotic, the ciprofloxacin was significantly less active (10-fold) in vitro against Giardia. However, potential for in vivo activity may exist, considering that the ciprofloxacin levels achievable in the gastrointestinal tract inhibit the proliferation of trophozoites (faecal concentrations range from 185 to $2200 \mathrm{mg} / 1$ following a 7-day course of CPX $500 \mathrm{mg}$ by the oral route every $12 \mathrm{~h}$ ) (Nord, 1988). In general, the fluoroquinolones have a broader spectrum of antimicrobial activity, are well tolerated and have an excellent safety record in long-term therapy (Segev et al., 1999). Therefore, ciprofloxacin may play an important role in the management of drug-resistant giardiasis or in patients with adverse reactions to other agents.

Another objective of this work was to clarify the drug action mechanism(s). The present study demonstrates that CPX inhibits the G. lamblia trophozoite attachment in vitro, induces morphological alterations and loss of cell viability. Our data showed a strong correlation between rupture of the plasma membrane and cell death induced by CPX. The main ultrastructural alterations promoted by CPX were ribosome disaggregation, presence of electron-dense blocks in the cytoplasm and nucleus, plasma membrane protrusions (blebs), membrane solubilisation and intracellular clearing. The dense blocks very likely correspond to coagulated proteins and/or products of degradation of ribosomes. Previous studies with bacteria support the relationship between membrane damage, ribosome disappearance and cell death (Silva et al., 1987; Niven et al., 1999). It has also been demonstrated that $\mathrm{Mg}^{2+}$, acting through stabilisation of the ribosome, is important for cell viability (Apirakaramwong et al., 1998). Considering these 
findings and that CPX had magnesium-chelating properties (Stahlmann and Lode, 1999), our results suggest an interaction of CPX and intracellular $\mathrm{Mg}^{2+}$ that could be the basis of CPX toxicity.

\section{References}

Aley, B.S., Zimmerman, M., Hetsko, M., Selsted, M.E., Gillin, F.D., 1994. Killing of Giardia lamblia by cryptdins and cationic neutrophil peptides. Infection and Immunity 62, 5397-5403.

Apirakaramwong, A., Fukuchi, J., Kashiwagi, K., Kakinuma, Y., Ito, E., Ishihama, A., Igarashi, K., 1998. Enhancement of cell death due to decrease in $\mathrm{Mg}^{2+}$ uptake by OmpC (cation-selective porin) deficiency in ribosome modulation factor-deficient mutant. Biochemical and Biophysical Research Communications 251, 482-487.

Barat, L.M., Bloland, 1997. Drug resistance among malaria and other parasites. Infectious Diseases of Clinics of North America 11, 969987.

Dougherty, T.J., Saukkonen, J.J., 1985. Membrane permeability changes associated with DNA gyrase inhibitors in Escherichia coli. Antimicrobial Agents and Chemotherapy 28, 200-206.

Edlind, T.D., Hang, T.L., 1990. Activity of the Anthelmintic benzimidazoles against Giardia lamblia in vitro. Journal of Infectious Diseases 162, 1408-1411.

Elliot, T.S.J., Selton, A., Greenwood, D., 1987. The response of Escherichia coli. to ciprofloxacin and norfloxacin. Journal of Medical Microbiology 23, 83-88.

Farthing, M.J.G., 1997. The molecular pathogenesis of giardiasis. Journal of Pediatric Gastroenterology and Nutrition 24, 79-88.

Hill, D.R., Pohl, R., Pearson, R.D., 1986. Giardia lamblia: a culture method for determining parasite viability. American Journal of Tropical Medicine and Hygiene 35, 1129-1133.

Ikerd, T.R., Koletar, S.L., 1993. In vitro activity of ciprofloxacin, temafloxacin, azitrhromycin, clarithromycin and metronidazole against Giardia lamblia. Journal of Antimicrobials and Chemotherapy 31, 615-617.

Keister, B.D., 1983. Axenic culture of Giardia lamblia in TYI-S-33 medium supplemented with bile. Transactions of the Royal Society of Tropical Medicine and Hygiene 77, 487-488.

Kidwai, M., Misra, P., Kumar, P., 1998. The fluorinated quinolones. Current Pharmaceutical Design 4, 101-118.

Kulda, J., Nohyhová, E., 1995. Giardia in humans and animals. In: Kreier, J.P. (ed.), Parasitic Protozoa, Academic Press, pp. 229-268.

Niven, G.W., Miles, C.A., Mackey, B.M., 1999. The effects of hydrostatic pressure on ribosome conformation in Echerichia coli: an in vivo study using differential scanning calorimetry. Microbiology $145,419-425$.

Nord, C.E., 1988. Effect of new quinolones on the human gastrointestinal microflora. Reviews of Infectious Diseases 10, S193-196.

Segev, S., Yaniv, I., Haverstock, D., Reinhart, H., 1999. Safety of longterm therapy with ciprofloxacin: data analysis of controlled clinical trials and review. Clinical Infection and Diseases 28, 299-308.

Silva, M.T., Appelberg, R., Silva, M.N.T., Macedo, P.M., (1987). In vivo killing and degradation of Mycobacterium aurum within mouse peritoneal macrophages. Infection and Immunity 55, 2006-2016.

Sousa, M.C., Poiares-da-Silva, J., 1999a. A new method for assessing metronidazole susceptibility of Giardia lamblia trophozoites. Antimicrobial Agents and Chemotherapy 43, 2939-2942.

Sousa, M.C., Poiares-da-Silva, J., 1999b. Cytotoxicity induced by bismuth subcitrate in Giardia lamblia trophozoites. Toxicology in Vitro 13, 591-598.

Stahlmann, R., Lode, H., 1999. Toxicity of quinolones. Drugs 58, 37-42. 